\title{
Exploring food effects on indinavir absorption with human intestinal fluids in the mouse intestine
}

Nico Holmstock ${ }^{\mathrm{a}}$, Tom De Bruyn ${ }^{\mathrm{a}}$, Jan Bevernage ${ }^{\mathrm{a}}$, Pieter Annaert ${ }^{\mathrm{a}}$, Raf Mols ${ }^{\mathrm{a}}$, Jan Tack $^{\mathrm{b}}$ and Patrick Augustijns ${ }^{\mathrm{a}}$

a Laboratory for Pharmacotechnology and Biopharmacy, KU Leuven, Campus Gasthuisberg, O\&N 2, Herestraat 49 box 921, B-3000 Leuven, Belgium.

${ }^{\mathrm{b}}$ Department of Gastroenterology, University Hospitals Leuven, O\&N, Herestraat 49 box 701, B-3000 Leuven, Belgium

nico.holmstock@pharm.kuleuven.be tom.debruyn@pharm.kuleuven.be jan.bevernage@pharm.kuleuven.be pieter.annaert@pharm.kuleuven.be raf.mols@pharm.kuleuven.be jan.tack@med.kuleuven.be patrick.augustijns@pharm.kuleuven.be

\section{Corresponding author:}

Patrick Augustijns

Laboratory for Pharmacotechnology and Biopharmacy, KU Leuven

Gasthuisberg O\&N 2 - Herestraat 49 box 921 - 3000 Leuven - Belgium

tel: +32-16-330301 - fax: +32-16-330305

e-mail: patrick.augustijns@ pharm.kuleuven.be 


\section{Keywords:}

Human intestinal fluids, mouse, in situ intestinal perfusion, food effects, indinavir 


\section{Introduction}

Food intake can alter the intestinal absorption of many orally administered drugs.

During early stage drug development, when the amount of available compound is limited, it is challenging to predict whether or not a drug candidate will undergo a food effect.

Nevertheless, a few methods can be used to predict whether the absorption of a drug candidate will be prone to food-drug interactions. Fleisher et al. demonstrated that a food effect can be anticipated based on the compound's solubility and permeability, as defined in the Biopharmaceutics Classification System (BCS) (Fleisher et al., 1999). Briefly, this method predicts that a high fat meal will increase the bioavailability of low solubility compounds (class II and IV) and decrease the AUC of BCS class III compounds, while no effect is anticipated for class I drugs. Physiologically based pharmacokinetic modeling can also be applied to establish food-drug interactions, but this method requires extensive information on physicochemical properties, intestinal permeability and clearance of the drug (Won et al., 2012). In later stages of drug development, dissolution data obtained in simulated intestinal fluids (Dressman and Reppas, 2000) and in vivo methods using dogs or other animals (Lentz, 2008) can be used to predict human fed and fasted pharmacokinetics.

The approach of using biorelevant media simulating the fasted and fed state (FaSSIF and FeSSIF, respectively) or even human intestinal fluids (HIF) aspirated in fasted and fed conditions may also be taken to explore food effects during early stage drug development in drug absorption assays such as Caco- 2 cell cultures. However, although the Caco-2 cell line is compatible with FaSSIF, it does not allow the use of FeSSIF or HIF because the integrity of the monolayer is not maintained during a sufficient period of time (Deferme et al., 2003; Ingels et al., 2002). Patel et al. created a modified version of FeSSIF by changing the salt composition and doubling the amount of lecithin, and showed that this modified FeSSIF was compatible with the Caco-2 model (Patel et al., 2006). Nevertheless, the question remains 
whether FaSSIF and FeSSIF are representative for FaHIF and FeHIF regarding their effect on intestinal drug absorption. Of all presently available absorption models, the in situ intestinal perfusion technique with mesenteric blood sampling is the closest one to the in vivo situation. Despite possible species differences in e.g. P-gp expression levels (Uchida et al., 2011), this technique has been shown to better predict drug absorption in humans as compared to cellbased assays (Salphati et al., 2001), which are only predictive for passively transported drugs (Artursson et al., 2001). Because of the presence of a protective mucus layer, this model is expected to be more compatible with SIF and HIF. However, to our knowledge, HIF have never been applied in this model.

A class of drugs which is very susceptible to food effects is the class of HIV protease inhibitors: food intake increases the AUC of saquinavir (+ 700\%), nelfinavir $(+200 \%$ to $300 \%)$, lopinavir/ritonavir (+ 48\% to $97 \%)$, atazanavir/ritonavir $(+35 \%)$ and darunavir/ritonavir (+ 30\%) (Boffito et al., 2005; Sekar et al., 2007). Since these are all BCS class II compounds, the food effect was correctly predicted with the method of Fleisher et al. For these class II compounds, food intake is expected to increase their solubility in the intraluminal environment (because of high concentrations of bile salts, phospholipids and lipid degradation products forming micelles), leading to a higher intestinal absorption. In contrast to the above mentioned HIV protease inhibitors, a negative food effect is observed for indinavir, also a BCS class II drug, for which the AUC decreases by $78 \%$ when administered with a high-fat meal (Yeh et al., 1998). These investigators proposed that this negative food effect may be attributed to a delayed gastric emptying and a possible precipitation of indinavir due to a higher gastric pH. However, Carver et al. (Carver et al., 1999) have shown that meals that leave the gastric $\mathrm{pH}$ unchanged also lower indinavir plasma concentrations, suggesting that other factors are playing a role in this food effect for indinavir. 
Therefore, for the first time, we used a novel combination of HIF in the mouse in situ intestinal perfusion technique with mesenteric blood sampling (Mols et al., 2009) and tested the hypothesis that a lower bioaccessible fraction of indinavir is responsible for a limited intestinal permeability in the fed state compared to the fasted state. 


\section{Materials and methods}

\subsection{Chemicals}

Indinavir sulfate was donated by Hetero Drugs Ltd (Hyderabad, India). Ritonavir (free base) was obtained from Xiamen Huasing Chemicals Co. (Xiamen Fujian, China). GF120918 (elacridar) was provided by GSK (London, UK). Stock solutions were prepared in DMSO. Ketamine (Anesketin) and xylazin (Xyl-M 2\%) were from Eurovet (Heusden, Belgium) and VMD (Arendonk, Belgium), respectively. Sodium acetate trihydrate and methanol were purchased from VWR International (Leuven, Belgium). Phosphate buffered saline (PBS) and Hanks' balanced salt solution (HBSS) were provided by Lonza (Basel, Switzerland). Sodium taurocholic acid practical grade was purchased from MP Biomedicals (Illkirch Cedex, France). Phospholipon 90G (lecithin) was from Nattermann Phospholipid Gmbh (Köln, Germany). All other reagents were used as supplied. Water was purified with a Maxima system (Elga Ltd., High Wycombe Bucks, UK). Ensure Plus (Abbott Laboratories B.V., Zwolle, The Netherlands) was used to simulate a standard meal. One portion of $200 \mathrm{~mL}$ has an energy content of $1.263 \mathrm{~kJ}$ of which lipids, carbohydrates and proteins constitute $29 \%$, $54 \%$ and $17 \%$ on energy basis, respectively; the osmolality amounts to $670 \mathrm{mOsm} / \mathrm{kg}$; the $\mathrm{pH}$ is 6.6.

\subsection{Media}

Transport medium consisted of HBSS buffered with HEPES (10 mM) to $\mathrm{pH} 7.4$. FaSSIF and FeSSIF were made according to the composition reported by Vertzoni et al. (revised standard FaSSIF and FeSSIF with practical grade taurocholate and soybean lecithin, Table 1) (Vertzoni et al., 2004). Human intestinal fluids (HIF) from the duodenum of four healthy volunteers (two female, two male, between 19 and 35 years old) were collected in two different nutritional states according to the method described by Bevernage et al. (Bevernage 
et al., 2011). The HIF were collected every $15 \mathrm{~min}$ for up to $120 \mathrm{~min}$ from the duodenum (D2-D3) after the intake of $200 \mathrm{~mL}$ of water (fasted state) or a liquid meal (Ensure Plus $400 \mathrm{~mL})+200 \mathrm{~mL}$ of water (fed state). For each nutritional state, one pooled sample was made by combining the aspirates from all 4 volunteers. The pooled HIF were stored at - 30 ${ }^{\circ} \mathrm{C}$ until further use. The characteristics of the intestinal fluids are shown in Table 2 and Table 3.

\subsection{Solubility measurements}

The thermodynamic solubility of indinavir sulfate was determined with the standard shake flask method in transport medium, FaSSIF, FeSSIF and in HIF of the fasted and fed state. All solubility experiments were performed in triplicate. Approximately $1 \mathrm{mg}$ of indinavir sulfate was added to microcentrifuge tubes containing $0.5 \mathrm{~mL}$ of the above mentioned media and placed in a prewarmed shaking incubator $\left[37^{\circ} \mathrm{C}\right.$ at $200 \mathrm{rpm}(\mathrm{KS} 4000 \mathrm{i}$ control incubator from Ika (Staufen, Germany)] for 24 hours. The solid phase was separated from the dissolved part using centrifugation $\left(15 \mathrm{~min}, 20.817 \mathrm{~g}\right.$ at $\left.37^{\circ} \mathrm{C}\right)$. The top layer was carefully removed by aspiration. The supernatant of the samples was diluted 1/50 with methanol:water $(50: 50 \mathrm{v} / \mathrm{v})$ and quantified using an HPLC system with UV detection.

\subsection{Media characterization}

\subsubsection{Viscosity measurements}

The viscosity of intestinal fluids was measured using the Vibro Viscometer SV-10 from A\&D Europe GMBH (Griesheim, Germany). $10 \mathrm{ml}$ of each solution was heated to $37^{\circ} \mathrm{C}$, after which the viscosity was measured.

\subsubsection{Lecithin measurements}


Phospholipid levels were measured in HIF using the Phospholipids FS kit with the Trulab L standard from Diagnostic Systems International (Holzheim, Germany).

\subsubsection{Osmolality measurements}

The osmolality of each intestinal medium was determined using the Osmometer model 3250 from Advanced Instruments, Inc. (Norwood, MA, USA).

\subsubsection{Bile salt measurements}

The individual bile salts were analyzed using the LC-MS/MS system described in section 2.7 according to an in-house validated method.

\subsection{In situ intestinal perfusion}

NMRI (Naval Medical Research Institute) mice were purchased from Janvier (Le Genest Saint Isle, France). Only male mice were used in the experiments. The experimental setup for mouse in situ intestinal perfusion experiments with mesenteric blood sampling has previously been described by Mols et al. (Mols et al., 2009). The ileum of the mouse was perfused. The perfusate consisted of transport medium or the respective (simulated or aspirated) intestinal media containing indinavir $(100 \mu \mathrm{M})$ in the absence or presence of ritonavir $(75 \mu \mathrm{M})$ and in the absence or presence of GF120918 $(4 \mu \mathrm{M})$. The perfusion experiments were performed using an open-loop set-up when using simulated intestinal fluids, whereas we opted for a closed-loop set-up when using HIF, since only a limited volume was available. In the open-loop setup, perfusate samples were taken at the beginning and the end of each experiment, and showed that a constant $\mathrm{C}_{\mathrm{donor}}$ was maintained during the experimental period. In the closed-loop setup, perfusate samples were taken every 10 minutes, and revealed no major changes in donor concentration. This could be attributed to the fact that the 
amount of indinavir which was absorbed into the mesenteric blood was very low compared to the total amount of indinavir present in the perfusate $(<1.5 \%)$. Blood samples were collected from the mesenteric vein for 60 min over 5 minute time intervals. Approval for the experiments with mice was granted by the Institutional Ethical Committee for Animal Experimentation of the KU Leuven.

\subsection{Dialysis}

We estimated the bioaccessible fraction of indinavir in every intestinal medium by performing dialysis using the HTD 96b from HTDialysis, LLC (Gales Ferry, CT, US). The donor and acceptor compartments were separated by cellulose membrane strips with a molecular cutoff weight of $12-14 \mathrm{kDa}(\approx 3 \mathrm{~nm})$, which are expected to be impermeable for micelles, since they were shown to be about $7 \mathrm{~nm}$ and $50 \mathrm{~nm}$ in diameter in FeSSIF and FaSSIF, respectively (Kloefer et al., 2010). The membranes were hydrated according to the manufacturer's instructions. The acceptor compartment was filled with $150 \mu \mathrm{L}$ of a phosphate buffer for which the osmolality and $\mathrm{pH}$ were equalized to this of the donor solution. This was done by preparing a concentrated phosphate buffer, which was subsequently diluted with water to the correct osmotic value; the $\mathrm{pH}$ was adjusted using diluted $\mathrm{NaOH}$ or $\mathrm{HCl}$. The donor compartment was loaded with an equal volume of intestinal medium containing indinavir $(100 \mu \mathrm{M})$ with ritonavir $(75 \mu \mathrm{M})$. Samples of the donor and acceptor compartment were taken after 1, 2 and 6 hours. Although this time frame was too short to reach equilibrium between donor and acceptor compartment, it allowed us to distinguish between the bioaccessible fractions in each medium. Samples from the donor and acceptor compartment were diluted 1:50 and 1:10 in methanol:water (50:50 v/v), respectively.

\subsection{Analysis}


Samples obtained from solubility and dialysis experiments were directly injected into the HPLC-UV system. The HPLC system consisted of a Waters Alliance 2695 separations module and a Nova-Pak ${ }^{\circledR}$ C18 Radial-Pak ${ }^{\mathrm{TM}}(4 \mu \mathrm{m}, 8$ x $100 \mathrm{~mm})$ column under radial compression (Waters, Milford, MA). UV absorption was monitored by a Waters absorbance detector (model no. W2487) at $260 \mathrm{~nm}$. The mobile phase consisted of $25 \mathrm{mM}$ sodium acetate $(\mathrm{pH} 5.5)$ and methanol $(25: 75 \mathrm{v} / \mathrm{v})$; the flow rate amounted to $1.2 \mathrm{ml} / \mathrm{min}$. The retention time of indinavir was $5.0 \mathrm{~min}$. After elution, the column was flushed with acetonitrile/water (90:10 v/v) for $1 \mathrm{~min}$ and re-equilibrated with mobile phase for $4 \mathrm{~min}$. The calibration curve was linear over the concentration range of 0.78 to $12.5 \mu \mathrm{M}$.

Quantifying indinavir in the blood samples required blood precipitation. $30 \mu \mathrm{L}$ of blood was diluted in $120 \mu \mathrm{L}$ of water, after which $300 \mu \mathrm{L}$ of acetonitrile was added. The samples were vortexed for 10 seconds and centrifuged at 20,817g for 5 minutes. The supernatant was injected into the LC-MS/MS. The Thermo Scientific (San Jose, USA) LC-MS/MS system comprised an Accela ${ }^{\circledR}$ autosampler, an Accela ${ }^{\circledR}$ pump and a TSQ Quantum Access ${ }^{\circledR}$ triple quadrupole mass spectrometer equipped with an electrospray ionisation (ESI) source. Data acquisition and peak integration were performed with the Xcalibur ${ }^{\circledR}$ 2.0.7 and LCquan ${ }^{\circledR}$ 2.5.6 software packages, respectively. A Kintetex ${ }^{\circledR} \mathrm{C} 18$ column $(50 \mathrm{~mm} \times 3.0 \mathrm{~mm}, 1.7 \mu \mathrm{m})$ (Phenomenex, The Netherlands), protected by a Krudkatcher Ultra HPLC In-Line filter (Phenomenex, The Netherlands) was used for chromatographic separation. The mobile phase consisted of a $0.5 \mathrm{mM}$ ammonium acetate buffer (pH 3.5) (A) and methanol (B). Gradient elution at a constant flow rate of $400 \mu \mathrm{L} / \mathrm{min}$ was performed as follows: $95 \%$ A decreased linearly to $5 \% \mathrm{~A}$ in $2 \mathrm{~min}$; constant flow of $5 \%$ A for $1 \mathrm{~min}$; linear increase back to $95 \% \mathrm{~A}$ in $10 \mathrm{sec}$; re-equilibration for 1.0 min with $95 \%$ A before the next injection. The total run time was $4.0 \mathrm{~min}$ and the injection volume was $10 \mu \mathrm{L}$ (partial loop mode). Under these conditions, the retention time of indinavir was $2.72 \mathrm{~min}$. The mass spectrometer was operated 
in the positive electrospray mode. The spray voltage was $3000 \mathrm{~V}$ and the capillary temperature was set at $275{ }^{\circ} \mathrm{C}$. The vaporizer temperature was $300{ }^{\circ} \mathrm{C}$. Nitrogen was used as the sheath gas (50 arbitrary units), ion sweep gas (10 arbitrary units) and auxiliary gas (20 arbitrary units). Argon was used as the collision gas at a pressure of $1.5 \mathrm{mTorr}$. The mass spectrometer was operated in a 3-channel selected reaction monitoring (SRM) mode. A single precursor-product ion pair was used for detection of indinavir: $\mathrm{m} / \mathrm{z} 614.5 \rightarrow 421.3$ (collision energy: $32 \mathrm{~V}$ ) with a scan time of $100 \mathrm{~ms}$. Both Q1 and Q3 were set at 0.7 unit mass resolution. An on-line motorized divert valve was used to introduce the eluent to the mass spectrometer over the period of $1.0-4.0 \mathrm{~min}$ for data acquisition. The eluent flow outside this interval was diverted to the waste. Calibration curves were linear over the concentration ranges of $31 \mathrm{nM}-5 \mu \mathrm{M}$. The assessment of intraday repeatability of indinavir, determined at $0.25 \mu \mathrm{M}$, resulted in a relative standard deviation of $6.0 \%(\mathrm{n}=6)$. The deviation from the theoretical concentration amounted to $+7.0 \%$. The interday precision (relative standard deviation; $\mathrm{n}=12$ ) of a standard of $1 \mu \mathrm{M}$ amounted to $6.2 \%$. The interday accuracy was $-6 \%$.

\subsection{Calculations}

The intestinal flux of indinavir was determined from the cumulative amount of indinavir appearing in the mesenteric blood between 40-60 minutes according to the following equation:

$$
\text { Flux }=\Delta \mathrm{Q} /(\Delta \mathrm{t} \times \mathrm{A})
$$

where $\mathrm{Q}$ is the cumulative amount of drug appearing in the mesenteric blood and $\mathrm{A}$ is the surface area of the perfused cylindrical intestinal segment.

\subsection{Statistics}


Statistical analysis was performed using an unpaired t-test as specified in the figure legends. 


\section{Results}

\subsection{Solubility of indinavir in intestinal fluids}

Preceding the intestinal absorption studies, we determined the thermodynamic solubility of indinavir in different media (Table 3). The solubility of indinavir in FaHIF was approximately 6-fold lower compared to that in FeHIF.

\subsection{System suitability}

Typical appearance profiles of indinavir in the mesenteric blood are shown as a function of time using the intestinal media FaSSIF (expected to be the least toxic medium due to the lowest amount of bile salts, lecithin and lipid degradation products) and FeHIF (expected to be the most toxic medium due to the high amount of bile salts, lecithin and lipid degradation products) (Figure 1). The linear cumulative transport of indinavir as a function of time indicates stable barrier functions during the entire duration of the perfusion experiments.

\subsection{Intestinal permeation of indinavir using different media}

We determined the intestinal permeation of indinavir dissolved in various media representative for the fasted and fed state, respectively, using the mouse in situ intestinal perfusion technique with mesenteric blood sampling (Figure 2). The data obtained in FaSSIF were adapted from (Holmstock et al., 2012). In order to exclude concentration-dependent effects, we used $100 \mu \mathrm{M}$ as a donor concentration. At this concentration, no precipitation of indinavir was observed in the perfusate, suggesting that supersaturated concentrations were being maintained during the experimental period, as observed for other drugs by Bevernage $e t$ al. (Bevernage et al., 2010). The permeation of indinavir was comparable using both simulated intestinal fluids (Flux $=4.2 \times 10^{-4} \mathrm{nmol} /\left({\mathrm{sec} \times \mathrm{cm}^{2}}^{2}\right)$ and $4.5 \times 10^{-4} \mathrm{nmol} /\left(\mathrm{sec} \times \mathrm{cm}^{2}\right)$ in FaSSIF and FeSSIF, respectively). However, when using HIF, the permeation of indinavir 
was 22-fold higher in FaHIF than in FeHIF (Flux $=11.3 \times 10^{-4} \mathrm{nmol} /\left(\mathrm{sec} \times \mathrm{cm}^{2}\right)$ and $0.5 \times 10^{-4}$ $\mathrm{nmol} /\left(\sec \times \mathrm{cm}^{2}\right)$, respectively). Inclusion of ritonavir $(75 \mu \mathrm{M})$ in the intestinal media increased the permeation of indinavir in FaSSIF 3-fold, while no effect was observed in FeSSIF. Similarly, adding ritonavir to HIF increased the permeation of indinavir in FaHIF 2fold, while this had no effect in FeHIF.

\subsection{Approximation of the free fraction of indinavir and ritonavir in intestinal fluids}

In order to estimate the free fraction of indinavir and ritonavir in fasted and fed state intestinal fluids, we performed dialysis experiments. Indinavir was spiked to each medium at a concentration of $100 \mu \mathrm{M}$, ritonavir at $75 \mu \mathrm{M}$. The concentrations of both drugs being measured in the acceptor compartment after 1, 2 and 6 hours are shown in Figure 3. When comparing the transport profiles of indinavir and ritonavir in the four media, it was striking that the appearance of both drugs in the FeHIF condition was extremely low as compared to the other media tested, indicating a very low bioaccessible fraction.

\subsection{Viscosity of intestinal fluids}

The viscosity values of the intestinal fluids are shown in Table $\mathbf{3}$ and were all comparable to the viscosity of water $(\approx 0.7 \mathrm{mPa} \times \mathrm{s})$. 


\section{Discussion}

Indinavir is an HIV protease inhibitor of the first generation. It is poorly soluble in water at physiological $\mathrm{pH}$, but because of its $\mathrm{pKa}$ values of 5.9 and 3.7, its solubility increases exponentially at a pH below 6 . Therefore, indinavir requires an acidic gastric $\mathrm{pH}$ to dissolve and become available for intestinal absorption (Lin et al., 1995). Indinavir absorption has been shown to be very susceptible to negative food effects (Yeh et al., 1998). Although these investigators hypothesized that an increased gastric $\mathrm{pH}$ causes a precipitation of indinavir, this was later disputed (Carver et al., 1999). Therefore, other factors may contribute to this negative food effect. We investigated the overall food effects on the intestinal absorption of indinavir using different intestinal media.

First of all, we observed a clear food effect on the solubility of indinavir. As intestinal fluids collected in the fed state contain various solubility enhancing compounds including bile salts, phospholipids and lipid degradation products forming mixed micelles, the solubilizing capacity of these fluids is larger as compared to transport medium. However, at the same time, this increased micellar entrapment of indinavir may potentially limit the free fraction available for absorption (bioaccessible fraction). To determine the bioaccessible fraction of indinavir in the different intestinal media, we performed dialysis experiments. The determination of the free concentration in a micellar system is a difficult task in view of the migration of monomers which may form micelles in the acceptor compartment. Dialysis experiments allowed us to compare differences in the bioaccessible fraction of indinavir between media in a relative way. The results of this approach appeared to be very reproducible. Already after two hours, the indinavir concentration in the acceptor compartment was more than 10-fold lower in the FeHIF condition compared to the FaHIF condition, suggesting a much lower bioaccessible fraction of indinavir in FeHIF compared to FaHIF. This lower bioaccessible fraction of indinavir resulted in a 22 -fold lower intestinal 
permeation in FeHIF compared to FaHIF when performing intestinal perfusion studies. These data show that an increased micellar entrapment of indinavir in the fed state may limit its intestinal absorption. This solubility/permeability interplay has been observed previously (Dahan et al., 2010; Katneni et al., 2006; Miller et al., 2011). The use of dialysis for elucidating the free drug fraction in biorelevant media has recently also been described by Frank et al. (Frank et al., 2012). In this study, it was assumed that the dialysis membrane is impermeable for micelles; therefore samples were taken when equilibrium was reached. However, in view of the migration of monomers which may form micelles in the acceptor compartment, determining the drug appearance rate in the acceptor compartment at 'preequilibrium' conditions may be more relevant for determining the free drug fraction.

Second, it is possible that the intestinal first-pass elimination of indinavir is affected by food, thereby possibly influencing intestinal metabolism and efflux. Indinavir is extensively metabolized by CYP3A4; however, intestinal first-pass metabolism for indinavir has been estimated to be only 5\% in humans (Lin, 1999), which excludes a food effect on intestinal metabolism. Furthermore, previous research in our laboratory has shown that the intestinal metabolism of indinavir in mice is negligible (Holmstock et al., 2012). A food effect involving P-gp may also play a role since indinavir is a substrate of P-gp, especially given the low bioaccessible fraction of indinavir in the fed state due to micellar entrapment and the reported $\mathrm{K}_{\mathrm{m}}$ of 40-60 $\mu \mathrm{M}$ (Lin, 1999). This may also explain why ritonavir, a known P-gp inhibitor, overrules the food restriction when it is co-administered with indinavir (Boyd, 2007). In our experiments, the presence of ritonavir increased the intestinal permeation of indinavir 2-fold when using FaHIF, while there was no increase when using FeHIF. This absence of P-gp inhibition by ritonavir in FeHIF may be explained by a very low bioaccessible fraction of ritonavir (Figure 3). As ritonavir is entrapped in the micelles, it cannot exert an inhibiting effect on P-gp. Therefore, one should differentiate between a 
possible direct and indirect effect of ritonavir on P-gp. Our experimental setup only allowed exploring a direct P-gp inhibitory effect, thereby mimicking a single dose administration. However, in clinical practice, twice daily dosing of indinavir/ritonavir results in steady state plasma concentrations of ritonavir (Saah et al., 2001), thereby potentially generating inhibiting concentrations at the level of intestinal P-gp (indirect effect).

Another factor which may contribute to the observed food effect is the difference in viscosity between FaHIF and FeHIF (Radwan et al., 2012). Because its effect on drug permeability remains unclear, we investigated whether a difference in viscosity between FaHIF and FeHIF could also explain the different intestinal permeation of indinavir. The viscosity of FaHIF and FeHIF was in the same range as plain water, which was expected since FeHIF was collected after administration of a liquid meal. Therefore, in our experimental setup, the observed difference in indinavir permeability between FaHIF and FeHIF could not be explained by a difference in viscosity. However, intake of a solid meal may result in a different outcome.

The predictive value of simulated intestinal fluids towards food effects was investigated as well. When using either FaSSIF or FeSSIF, a comparable intestinal permeation of indinavir was observed, corresponding with a similar bioaccessible fraction of indinavir in the two media. Ritonavir caused an increase in indinavir permeation only in FaSSIF, but not in FeSSIF. This may be explained by a lower bioaccessible fraction of ritonavir in FeSSIF compared to FaSSIF. These findings indicate that simulated intestinal fluids used in fooddrug interaction studies do not always provide the same insights as when using HIF. Characterization of HIF will be the subject of further studies, which may allow correlating individual differences in medium characteristics to the observed food effects. Finally, these factors can then be implemented in absorption models in order to allow easy screening for food effects. 


\section{Conclusion}

Our results indicate that, in the fed state, the bioaccessible fraction of indinavir in the intestine is low due to micellar entrapment, which significantly reduces its intestinal absorption. Because of this low bioaccessible fraction of indinavir, a saturation of intestinal P-gp is unlikely, meaning that P-gp may significantly limit the oral bioavailability of indinavir in vivo. This contrasts to the fasted state, where a higher bioaccessible fraction of indinavir resulted in a high intestinal permeation. Steady-state plasma concentrations of ritonavir may inhibit P-gp mediated efflux of indinavir in the fed state, thereby increasing its intestinal absorption. However, this remains to be investigated. Finally, we conclude that, using HIF (FaHIF and FeHIF) as a solvent system for the first time, the in situ intestinal perfusion technique allowed us to gather mechanistic insights on the effects of food on indinavir absorption.

\section{Acknowledgements}

This research was funded by grants from: (1) Institute for the Promotion of Innovation through Science and Technology in Flanders (IWT), (2) Fund for Scientific Research in Flanders (FWO), and (3) 'Onderzoeksfonds' of the KU Leuven in Belgium. 


\section{References}

Artursson, P., Palm, K., Luthman, K., 2001. Caco-2 monolayers in experimental and theoretical predictions of drug transport. Adv. Drug Deliv. Rev. 46, 27-43.

Bevernage, J., Brouwers, J., Clarysse, S., Vertzoni, M., Tack, J., Annaert, P., Augustijns, P., 2010. Drug supersaturation in simulated and human intestinal fluids representing different nutritional states. J Pharm Sci. 99, 4525-4534.

Bevernage, J., Forier, T., Brouwers, J., Tack, J., Annaert, P., Augustijns, P., 2011. Excipientmediated supersaturation stabilization in human intestinal fluids. Mol. Pharm. 8, 564570.

Boffito, M., Acosta, E., Burger, D., Fletcher, C.V., Flexner, C., Garaffo, R., Gatti, G., Kurowski, M., Perno, C.F., Peytavin, G., Regazzi, M., Back, D., 2005. Current status and future prospects of therapeutic drug monitoring and applied clinical pharmacology in antiretroviral therapy. Antivir. Ther. (Lond.). 10, 375-392.

Boyd, M., 2007. Indinavir: the forgotten HIV-protease inhibitor. Does it still have a role? Expert Opin Pharmacother. 8, 957-964.

Carver, P.L., Fleisher, D., Zhou, S.Y., Kaul, D., Kazanjian, P., Li, C., 1999. Meal composition effects on the oral bioavailability of indinavir in HIV-infected patients. Pharm. Res. 16, 718-724.

Dahan, A., Miller, J.M., Hoffman, A., Amidon, G.E., Amidon, G.L., 2010. The solubilitypermeability interplay in using cyclodextrins as pharmaceutical solubilizers: mechanistic modeling and application to progesterone. J Pharm Sci. 99, 2739-2749.

Deferme, S., Tack, J., Lammert, F., Augustijns, P., 2003. P-glycoprotein attenuating effect of human intestinal fluid. Pharm. Res. 20, 900-903.

Dressman, J.B., Reppas, C., 2000. In vitro-in vivo correlations for lipophilic, poorly watersoluble drugs. Eur J Pharm Sci. 11 Suppl 2, S73-80. 
Fleisher, D., Li, C., Zhou, Y., Pao, L.H., Karim, A., 1999. Drug, meal and formulation interactions influencing drug absorption after oral administration. Clinical implications. Clin Pharmacokinet. 36, 233-254.

Frank, K.J., Westedt, U., Rosenblatt, K.M., Hölig, P., Rosenberg, J., Mägerlein, M., Brandl, M., Fricker, G., 2012. Impact of FaSSIF on the solubility and dissolution-/permeation rate of a poorly water-soluble compound. Eur J Pharm Sci. 47, 16-20.

Holmstock, N., Annaert, P., Augustijns, P., 2012. Boosting of HIV Protease Inhibitors by Ritonavir in the Intestine: The Relative Role of Cytochrome P450 and P-Glycoprotein Inhibition Based on Caco-2 Monolayers versus In Situ Intestinal Perfusion in Mice. Drug Metab. Dispos. 40, 1473-1477.

Ingels, F., Deferme, S., Destexhe, E., Oth, M., Van den Mooter, G., Augustijns, P., 2002. Simulated intestinal fluid as transport medium in the Caco-2 cell culture model. Int J Pharm. 232, 183-192.

Katneni, K., Charman, S.A., Porter, C.J.H., 2006. Permeability assessment of poorly watersoluble compounds under solubilizing conditions: the reciprocal permeability approach. J Pharm Sci. 95, 2170-2185.

Kloefer, B., Van Hoogevest, P., Moloney, R., Kuentz, M., Leigh, M.L.S., Dressman, J., 2010. Study of a Standardized Taurocholate- Lecithin Powder for Preparing the Biorelevant Media FeSSIF and FaSSIF. Dissolution Technologies. 17, 6-13.

Lentz, K.A., 2008. Current methods for predicting human food effect. AAPS J. 10, 282-288. Lin, 1999. Role of pharmacokinetics in the discovery and development of indinavir. Adv. Drug Deliv. Rev. 39, 33-49.

Lin, J.H., Chen, I.W., Vastag, K.J., Ostovic, D., 1995. pH-dependent oral absorption of L735,524, a potent HIV protease inhibitor, in rats and dogs. Drug Metab. Dispos. 23, $730-735$. 
Miller, J.M., Beig, A., Krieg, B.J., Carr, R.A., Borchardt, T.B., Amidon, G.E., Amidon, G.L., Dahan, A., 2011. The solubility-permeability interplay: mechanistic modeling and predictive application of the impact of micellar solubilization on intestinal permeation. Mol. Pharm. 8, 1848-1856.

Mols, R., Brouwers, J., Schinkel, A.H., Annaert, P., Augustijns, P., 2009. Intestinal perfusion with mesenteric blood sampling in wild-type and knockout mice: evaluation of a novel tool in biopharmaceutical drug profiling. Drug Metab. Dispos. 37, 1334-1337.

Patel, N., Forbes, B., Eskola, S., Murray, J., 2006. Use of simulated intestinal fluids with Caco-2 cells and rat ileum. Drug Dev Ind Pharm. 32, 151-161.

Radwan, A., Amidon, G.L., Langguth, P., 2012. Mechanistic investigation of food effect on disintegration and dissolution of BCS class III compound solid formulations: the importance of viscosity. Biopharm Drug Dispos. 33, 403-416.

Saah, A.J., Winchell, G.A., Nessly, M.L., Seniuk, M.A., Rhodes, R.R., Deutsch, P.J., 2001. Pharmacokinetic profile and tolerability of indinavir-ritonavir combinations in healthy volunteers. Antimicrob. Agents Chemother. 45, 2710-2715.

Salphati, L., Childers, K., Pan, L., Tsutsui, K., Takahashi, L., 2001. Evaluation of a singlepass intestinal-perfusion method in rat for the prediction of absorption in man. J. Pharm. Pharmacol. 53, 1007-1013.

Sekar, V., Kestens, D., Spinosa-Guzman, S., De Pauw, M., De Paepe, E., Vangeneugden, T., Lefebvre, E., Hoetelmans, R.M.W., 2007. The effect of different meal types on the pharmacokinetics of darunavir (TMC114)/ritonavir in HIV-negative healthy volunteers. J Clin Pharmacol. 47, 479-484.

Uchida, Y., Ohtsuki, S., Katsukura, Y., Ikeda, C., Suzuki, T., Kamiie, J., Terasaki, T., 2011. Quantitative targeted absolute proteomics of human blood-brain barrier transporters and receptors. J. Neurochem. 117, 333-345. 
Vertzoni, M., Fotaki, N., Kostewicz, E., Stippler, E., Leuner, C., Nicolaides, E., Dressman, J., Reppas, C., 2004. Dissolution media simulating the intralumenal composition of the small intestine: physiological issues and practical aspects. J. Pharm. Pharmacol. 56, $453-462$.

Won, C.S., Oberlies, N.H., Paine, M.F., 2012. Mechanisms underlying food-drug interactions: Inhibition of intestinal metabolism and transport. Pharmacol. Ther.

Yeh, K.C., Deutsch, P.J., Haddix, H., Hesney, M., Hoagland, V., Ju, W.D., Justice, S.J., Osborne, B., Sterrett, A.T., Stone, J.A., Woolf, E., Waldman, S., 1998. Single-dose pharmacokinetics of indinavir and the effect of food. Antimicrob. Agents Chemother. $42,332-338$. 


\section{Figure legends}

Figure 1: The cumulative amount on indinavir appearing in the mesenteric blood as a function of time using the mouse in situ intestinal perfusion model. The ileum of NMRI mice was perfused with indinavir $(100 \mu \mathrm{M})$ in $\operatorname{FaSSIF}(\mathbf{\bullet})$ and FeHIF $(\bullet)$. Values, which were corrected for the surface area of the intestinal segment, show the mean $\pm \operatorname{SD}(n=3)$.

Figure 2: The flux of indinavir $\left(\mathrm{C}_{\mathrm{donor}}=100 \mu \mathrm{M}\right)$ over the mouse ileum using different media in the absence (open bars) and presence of the P-gp inhibitor GF120918 (4 $\mu \mathrm{M})$ (light gray bars) and ritonavir $(75 \mu \mathrm{M})$ (dark gray bars). Bars represent the mean $\pm \operatorname{SD}(n=3)$. significantly different from control condition $(\mathrm{p}<0.05)$. Statistical significance between the control and P-gp inhibitory condition was evaluated using an unpaired t-test. The data obtained in FaSSIF were adapted from (Holmstock et al., 2012).

Figure 3: Indinavir (A) and ritonavir (B) concentrations measured in the acceptor compartment as a function of time in the dialysis setup. Donor concentrations were $100 \mu \mathrm{M}$ for indinavir and $75 \mu \mathrm{M}$ for ritonavir in $\operatorname{FaSSIF}(\bullet), \operatorname{FeSSIF}(\bullet), \operatorname{FaHIF}(\circ)$ and FeHIF ( $\square$ ). The acceptor medium consisted of buffer for which the osmolality and $\mathrm{pH}$ was equalized to this of the donor solution. Values are shown as the mean $\pm \operatorname{SD}(n=3)$. 
Figure 1

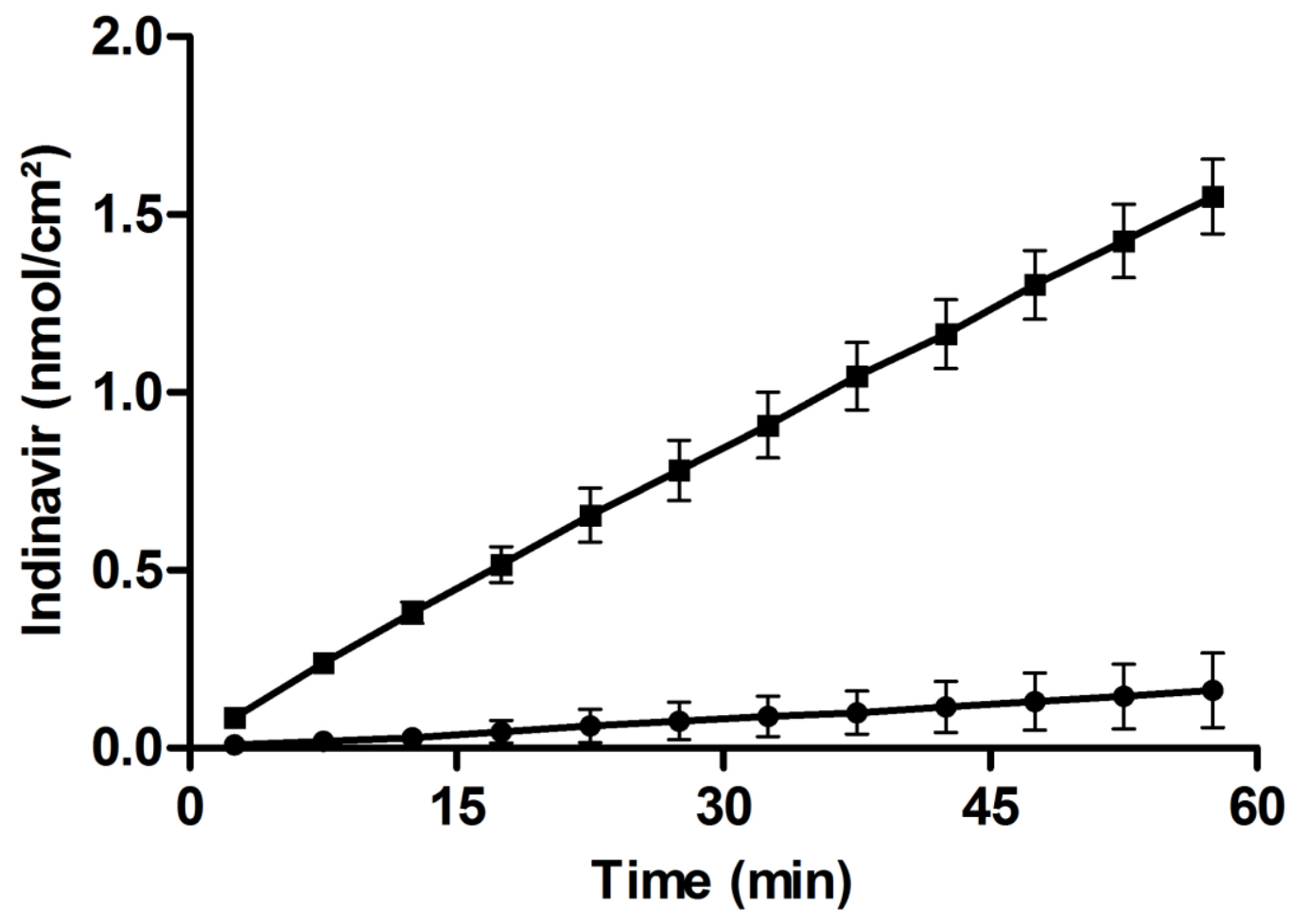


Figure 2

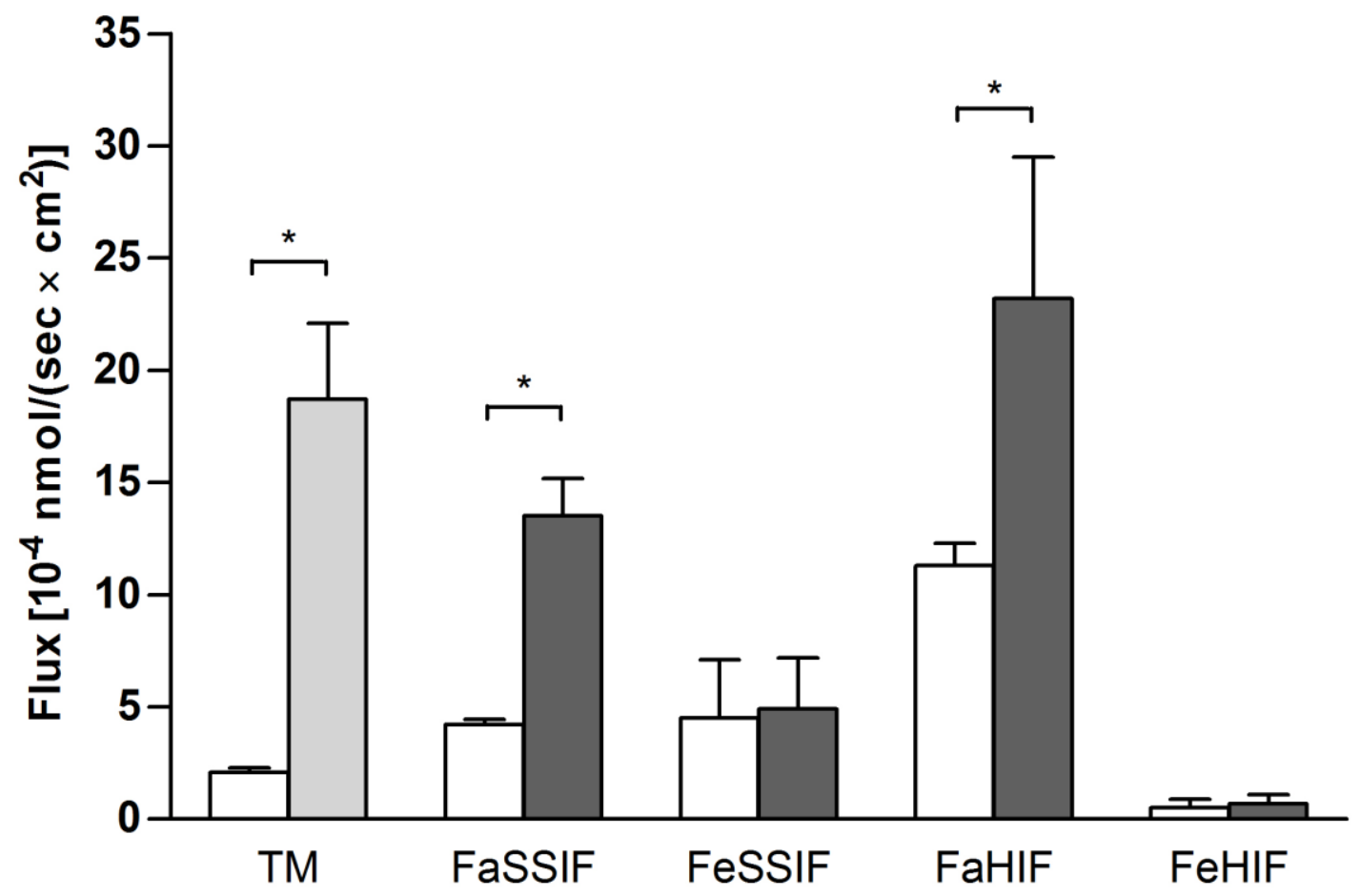


Figure 3

A

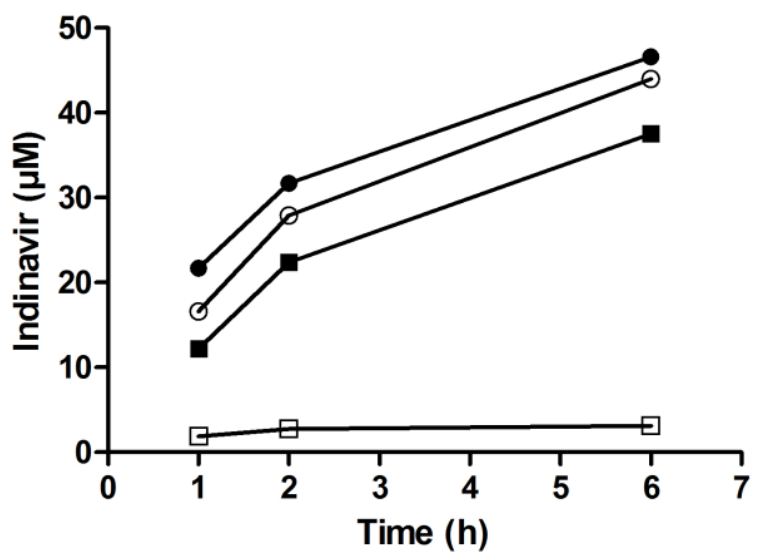

B

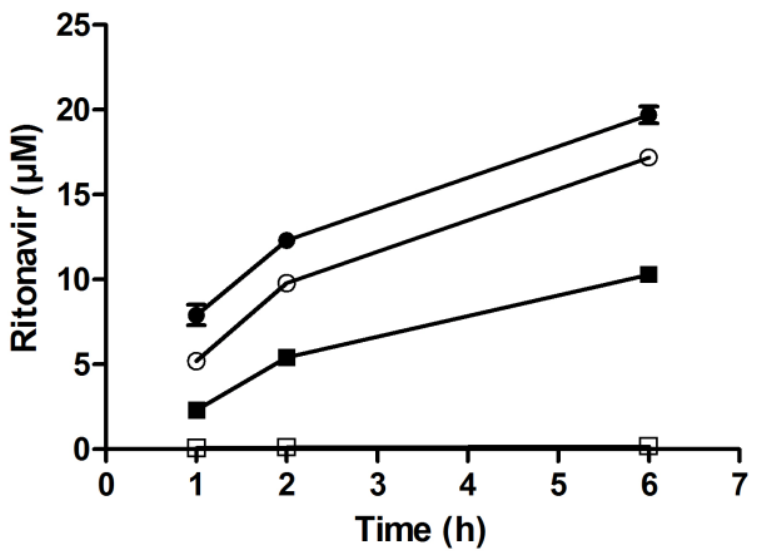


Table 1: Composition of FaSSIF and FeSSIF.

\begin{tabular}{lll}
\hline Component & FaSSIF & FeSSIF \\
\hline Sodium taurocholate (NaTC) & $3 \mathrm{mM}$ & $15 \mathrm{mM}$ \\
Lecithin & $0.75 \mathrm{mM}$ & $3.75 \mathrm{mM}$ \\
$\mathrm{NaH}_{2} \mathrm{PO}_{4} \cdot \mathrm{H}_{2} \mathrm{O}$ & $3.95 \mathrm{~g}$ & - \\
$\mathrm{Glacial}$ acetic acid & - & $8.65 \mathrm{~g}$ \\
$\mathrm{NaCl}$ & $6.19 \mathrm{~g}$ & $30.93 \mathrm{~g}$ \\
$\mathrm{NaOH}$ & $0.35 \mathrm{~g}$ & $1.74 \mathrm{~g}$ \\
$\mathrm{pH}$ & 6.5 & 5.0 \\
Water & $\mathrm{ad} 1 \mathrm{~L}$ & $\mathrm{ad} 1 \mathrm{~L}$ \\
\hline
\end{tabular}


Table 2: Individual bile salt and lecithin concentrations in FaHIF and FeHIF.

\begin{tabular}{cccccccccccc}
\hline \multirow{2}{*}{ Medium } & \multicolumn{9}{c}{ Bile Salt Concentration (mM) } & \multicolumn{3}{c}{\begin{tabular}{c} 
Lecithin \\
\cline { 2 - 8 }
\end{tabular}} & TUDC & GUDC & TC & GC & TCDC & TDC & GCDC & GDC & Total & $(\mathrm{mM})$ \\
\hline FaHIF & 0.045 & 0.244 & 0.768 & 2.766 & 0.622 & 0.381 & 2.281 & 1.016 & 8.125 & 2.06 \\
FeHIF & 0.057 & 0.232 & 0.789 & 2.585 & 0.748 & 0.383 & 2.371 & 1.250 & 8.415 & 5.81 \\
\hline
\end{tabular}

TUDC: tauroursodeoxycholate,

GUDC: glycoursodeoxycholate,

TC: taurocholate,

GC: glycocholate,

TCDC: taurochenodeoxycholate,

TDC: taurodeoxycholate,

GCDC: glycochenodeoxycholate,

GDC: glycodeoxycholate 
Table 3: Characteristics of the different media with respect to $\mathrm{pH}$, osmolality, viscosity and the thermodynamic solubility of indinavir.

\begin{tabular}{lcccc}
\hline Medium & $\mathrm{pH}$ & $\begin{array}{c}\text { Osmolality } \\
(\mathrm{mOsm} / \mathrm{kg})\end{array}$ & $\begin{array}{c}\text { Viscosity } \\
(\mathrm{mPa} \times \mathrm{s})\end{array}$ & $\begin{array}{c}\text { Solubility indinavir } \\
(\mu \mathrm{M})\end{array}$ \\
\hline TM & 7.4 & 284 & 0.7 & $61 \pm 3$ \\
FaSSIF & 6.5 & 264 & 0.7 & $79 \pm 1$ \\
FeSSIF & 5 & 649 & 0.7 & $2214 \pm 196$ \\
FaHIF & 7.5 & 224 & 0.65 & $84 \pm 3$ \\
FeHIF & 6 & 379 & 1.30 & $517 \pm 94$ \\
\hline
\end{tabular}

